\title{
Criminologie
}

\section{Les étapes de la rééducation : formalisation et vérification}

\section{Marc LeBlanc et Bernard Tessier}

Volume 11, numéro 1, 1978

Centres d’accueil du Québec : accréditation et évaluation

URI : https://id.erudit.org/iderudit/017081ar

DOI : https://doi.org/10.7202/017081ar

Aller au sommaire du numéro

Éditeur(s)

Les Presses de l'Université de Montréal

ISSN

0316-0041 (imprimé)

1492-1367 (numérique)

Découvrir la revue

Citer cet article

LeBlanc, M. \& Tessier, B. (1978). Les étapes de la rééducation : formalisation et vérification. Criminologie, 11(1), 24-45. https://doi.org/10.7202/017081ar d'utilisation que vous pouvez consulter en ligne.

https://apropos.erudit.org/fr/usagers/politique-dutilisation/ 


\section{LES ETAPES DE LA REÉDUCATION : FORMALISATION ET VÉRIFICATION *}

Marc LeBlanc

Bernard Tessier

Une innovation majeure dans le domaine de la rééducation, comme le montre Cusson (1974), est le développement, par Boscoville et ses animateurs, de la théorie des étapes de la rééducation qui permet d'orienter et de contrôler l'évolution du jeune délinquant pendant le processus de rééducation. Si l'idée d'étapes de rééducation avait été perçue par Makarenko, Aichhorn, Redl et Bettelheim, il en revient à Gilles Gendreau et à ses collaborateurs de Boscoville de l'avoir concrétisée et appliquée et à Jeannine Guindon (1970) de l'avoir formalisée en théorie.

Cet article, dans la lignée de ces entreprises, traite de la question des indicateurs d'étapes de la rééducation. 11 comprendra trois parties : la présentation de la théorie des étapes et leur définition, l'opérationnalisation de cette théorie et sa vérification dans les domaines des activités, des relations avec les pairs et des relations avec les éducateurs.

\section{LA THÉORIE DES ETAPES}

La théorie des étapes, comme toute théorie du développement, postule un passage graduel d'un état d'équilibre rudimentaire à un état d'équilibre supérieur. Et comme le montre Guindon (1970), le processus de développement n'est pas une croissance quantitative continue mais plutôt une suite de phases discontinues et qualitativement distinctes. Chaque phase, chaque étape est un stade, un nouvel équilibre ; en fait, chaque étape est une structure d'ensemble qui découle logiquement de la précédente.

Guindon (1970) nous dit qu'à chaque étape, l'organisation du moi est remise en cause sur les plans de la configuration du passé et du futur, des capacités de se confronter avec de nouvelles tâches et situations, de la combinaison des pulsions et défenses et du rayon des rencontres significatives.

* Cet article s'inscrit dans le cadre d'une évaluation de Boscoville pour le Groupe de recherche sur l'inadaptation juvénile de l'Université de Montréal et subventionnée par : ministère des Affaires sociales, ministère du Solliciteur général, ministère de la Santé et du Bien-être et les fondations annexes. 
Conformément à ce cadre théorique, le processus de rééducation devient une démarche d'acquisition de l'autonomie (Tessier, 1974), et les étapes du processus de rééducation peuvent être conçues comme un ensemble de buts à court terme qui sont proposés aux jeunes afin de les faire participer progressivement à leur rééducation (Cusson, 1974). Le processus de rééducation, tel que véçu à Boscoville, devient donc un modèle d'évolution en quatre étapes : acclimatation, contrôle, production et personnalité (Gendreau, 1964 ; Guindon, 1970).

\section{L'étape acclimatation}

À l'étape acclimatation, on y propose un programme d'action dont le but principal est d'apprendre à utiliser les divers moyens mis à la disposition du jeune pour lui permettre d'atteindre, à brève échéance, un but proposé.

Il faut comprendre le terme "moyen » dans un sens très large. Il s'applique aussi bien aux personnes, éducateurs et pairs, présentes dans les diverses situations de vie, qu'aux instruments ou aux procédures à utiliser; de même qu'au temps et à l'espace mis à la disposition du jeune.

La démarche d'apprentissage est graduée de façon à proposer des défis essentiellement surmontables. C'est ainsi que dans les trois premières phases, les buts à atteindre sont proposés par le programme et que la démarche d'autonomie est particulièrement orientée sur la maîtrise des moyens, leur sélection et la planification de leur utilisation.

A la première étape, on commence par acclimater le jeune à l'institution, à lui faire accepter le fait que Boscoville est un milieu de traitement. On doit faire admettre au jeune qu'il a besoin d'aide et que Boscoville peut répondre à ce besoin (Tessier, 1970). Par la suite, il convient de faire en sorte que le jeune soit dans l'impossibilité de continuer son agir délinquant pour qu'il puisse vivre en harmonie avec le milieu (Cusson, 1974). Finalement, le jeune doit faire l'expérience d'une réussite pour lui permettre de faire le lien entre ce qu'il fait et les conséquences de son action (Guindon, 1970).

\section{L'étape contrôle}

Alors qu'à l'étape acclimatation on maîtrise l'utilisation ou le maniement des divers moyens, à l'étape contrôle on propose 
une démarche d'apprentissage de sélection de moyens appropriés pour atteindre un but proposé, tout en prenant conscience du rôle personnel joué dans la production des résultats atteints.

En corollaire de cet objectif, le jeune devra apprendre à respecter le milieu où il se trouve, ce qui exigera un effort de conformité envers les réalités du milieu (Tessier, 1970). Il devra aussi s'intégrer au groupe grâce à une maîtrise de ses actes et à une capacité de répondre aux attentes des autres membres du groupe. Cette intégration assure les conditions nécessaires à l'intériorisation progressive des contrôles personnels (Guindon, 1970).

Le jeune aura atteint le stade du contrôle dans la mesure où les acquisitions suivantes seront consolidées : comportement en accord avec la tradition rééducative du milieu et les mours du groupe, sélection de moyens adéquats pour atteindre les buts des diverses activités, perception de l'éducateur comme une autorité sécurisante.

\section{L'étape production}

Maintenant que le jeune peut choisir judicieusement les moyens appropriés pour atteindre un but, il s'engagera dans l'apprentissage de la planification à longue échéance pour la poursuite des buts proposés, tout en tenant compte de l'ensemble des éléments personnels et sociaux qui constituent son expérience de vie totale.

Le jeune de l'étape production acquiert donc une méthode de travail, il utilise le milieu et ses ressources pour atteindre des résultats concrets. La découverte de sa compétence et du sentiment d'efficacité permettra donc au jeune d'envisager son avenir. Cette étape est aussi marquée par le rapprochement du jeune et de l'éducateur et le choix d'amis qui ont les mêmes orientations et aspirations (Cusson, 1974). L'éducateur devient alors un ami, un homme dont on admire la compétence ; celui avec qui l'on pourra s'identifier.

\section{L'étape personnalité}

A l'étape personnalité, le jeune réalise et accepte qu'il n'est plus et ne sera jamais le même que celui qui arrivait à l'institution (Tessier, 1970). Maintenant, il a la maîtrise de lui-même et de l'environnement. Il peut se servir de sa capacité d'utiliser, 
de sélectionner et de planifier en fonction de l'exercice d'une créativité à tous les niveaux dans la poursuite des buts proposés ou personnels, il devient maintenant capable de créer des moyens nouveaux à partir des acquis déjà réalisés ; ou bien d'appliquer les acquisitions préalables à des situations nouvelles. En même temps, la sélection des buts à poursuivre relèvera de son entière responsabilité et son choix sera essentiellement guidé par ses options de vie personnelle. Pendant cette période, l'accent est mis sur les options fondamentales, les valeurs et l'avenir (Cusson, 1974).

Les théories des étapes du processus de rééducation proposent donc un modèle d'action centré sur des buts généraux atteignables à travers un cheminement individualisé. Voyons maintenant comment cette théorie peut nous permettre de formuler des indicateurs d'étapes qui permettront de situer le jeune dans son évolution pendant la rééducation.

\section{LES INDICATEURS D'ÉTAPES}

L'opérationalisation de la théorie des étapes exigeait au préalable la solution de certains problèmes : dans quelles situations de vie va-t-on observer l'évolution des jeunes? Chaque étape est-elle un tout indissociable ? Quelles seront les caractéristiques que doivent posséder les indicateurs d'étapes?

La conceptualisation originale des étapes proposait, comme le montre Tessier (1971), son application en situation d'activités spécialement choisies et organisées pour les diverses catégories d'utilisateurs. Toutefois la tradition d'utilisation à Boscoville veut que l'évaluation des jeunes se fasse dans trois principales situations de vie : en activités, dans les relations avec les éducateurs et dans les relations avec les pairs. En conséquence, nous avons recherché des indicateurs qui se rapportent à ces trois situations de vie.

La recherche d'indicateurs d'étapes doit, par ailleurs, être guidée par la constatation que chaque étape n'est pas un tout indissociable. En effet, Guindon (1970) affirme que chaque étape comporte une période initiale de préparation et une période finale de rendement. Toutefois la tradition d'utilisation suppose un cheminement progressif ; ainsi, chaque étape devrait être subdivisée en trois phases qui impliquent des acquisitions consécutives : la phase initiale ou de déclenchement, la phase intermédiaire ou 
d'organisation et la phase finale ou de consolidation. Compte tenu de ce critère, le processus de rééducation apparaît comme un modèle d'évolution en douze phases regroupées en quatres étapes. Ce modèle spécifie que chaque phase représente la poursuite d'acquisitions, d'objectifs à court terme ; celles-ci se feront dans un ordre consécutif et, de plus, il y aura conservation des acquis suivant la théorie de base. L'escalier représente très bien ce modèle où les acquisitions se font progressivement tout en étant cumulatives.

Puisque nous avons justifié le fait que les indicateurs d'étapes doivent se rapporter à des situations de vie et à des phases d'évolution, les indicateurs devront se rapporter à chacune des cases du schéma suivant :

\begin{tabular}{|c|c|c|c|c|c|c|c|c|c|c|c|c|}
\hline Etapes & \multicolumn{3}{|c|}{ Acclimatation } & \multicolumn{3}{|c|}{ Contrôle } & \multicolumn{3}{|c|}{ Production } & \multicolumn{3}{|c|}{ Personnalité } \\
\hline $\begin{array}{l}\text { Situation } \\
\text { de vie } \\
\text { Phases }\end{array}$ & $1^{1}$ & $2^{2}$ & $3^{3}$ & 1 & 2 & 3 & 1 & 2 & 3 & 1 & 2 & 3 \\
\hline Activités & 1 & 2 & 3 & 4 & 5 & 6 & 7 & 8 & 9 & 10 & 11 & 12 \\
\hline Educateurs & 1 & 2 & 3 & 4 & 5 & 6 & 7 & 8 & 9 & 10 & 11 & 12 \\
\hline Pairs & 1 & 2 & 3 & 4 & 5 & 6 & 7 & 8 & 9 & 10 & 11 & 12 \\
\hline
\end{tabular}

1. phase initiale ou de déclenchement

2. phase intermédiaire ou d'organisation

3. phase finale ou de consolidation

Ayant déterminé les indicateurs qui nous sont nécessaires pour mesurer, selon l'évolution du jeune, le processus de rééducation, voyons maintenant quelles devront être les caractéristiques de ces indicateurs d'étapes.

Chaque indicateur devra remplir trois conditions :

- permettre de situer rapidement le niveau d'évolution des jeunes à l'intérieur du processus de rééducation ;

- décrire les conduites observables à chacune des douze phases du processus de rééducation et ce, dans trois situations de vie ;

- rendre possible une évaluation, un jugement d'ensemble, plutôt qu'une observation clinique directe et limitée dans le temps. 
Si nous optons pour des indicateurs qui permettent de situer rapidement le niveau d'évolution des jeunes, c'est en raison d'un objectif d'application. En effet, si nous voulons que cet instrument ne soit pas seulement un instrument de recherche mais en devienne un d'action, il lui faut ce caractère de rapidité car les éducateurs doivent évaluer régulièrement une quinzaine de jeunes.

Les indicateurs doivent constituer des conduites observables parce qu'une des réalités fondamentales de la rééducation est cette tentative constante pour modifier le comportement du jeune. Le comportement référant à des conduites significatives, directement observables par les éducateurs et issues de la vie quotidienne du jeune à Boscoville. Ces conduites sont significatives parce qu'elles expriment des attitudes, des sentiments; ce sont des actions ayant une finalité quelconque. Par contre, ces conduites ne doivent pas faire appel à la subjectivité de l'éducateur, à la spéculation ou à l'arbitraire d'une interprétation ; elles doivent constituer des objets observables. Finalement, ces conduites doivent référer au vécu actuel sans se rapporter à quelque chose de situé momentanément dans l'espace et le temps.

Compte tenu de ces exigences pour la formulation des indicateurs et de la théorie qui parle de phases et d'étapes comme des stades, c'est-à-dire une structure ou une organisation spécifique d'éléments ${ }^{1}$, il s'ensuit que nous devons demander aux éducateurs un jugement d'ensemble sur la présence de cette structure. On ne peut donc pas demander aux éducateurs si les éléments sont présents à l'aide d'une liste de contrôle mais il faut plutôt demander aux éducateurs d'évaluer si la présence et l'organisation des éléments sont telles que l'on puisse situer un jeune à telle étape du processus de rééducation.

\section{L'INSTRUMENT ET LES DONNÉES}

Ayant établi les cadres de l'instrument, c'est-à-dire trois situations d'évaluation (relations avec les pairs, relations avec les éducateurs, activités) et 12 phases à transformer en indicateurs qui impliquent un jugement sur la présence d'une structure donnée, les indicateurs ont été identifiés et formulés grâce à la démarche suivante.

1. Cette définition de stade correspond à celle la plus fréquemment utilisée en psychologie génétique ; voir Wohlwill (1973). 
Premièrement, l'équipe ${ }^{2}$ a réalisé des entrevues avec des éducateurs et des responsables de Boscoville; ces entrevues avaient pour objectif l'identification des éléments devant constituer les indicateurs d'étapes. Ces données, complétées par l'expérience que l'équipe avait de Boscoville et de la rééducation et orientées à l'aide de la théorie, ont permis de formuler les 36 indicateurs d'étapes.

Deuxièmement, les indicateurs d'étapes ont été soumis à un comité d'experts ${ }^{3}$ qui a analysé la validité des indicateurs en regard de la théorie et qui s'est assuré que ceux-ci étaient formulés clairement et sans ambiguité.

Finalement, l'équipe a constitué la liste des 36 indicateurs d'étapes (12 pour les relations avec les éducateurs, 12 pour les relations avec les pairs, 12 pour les activités) ; cette liste est présentée en annexe.

L'instrument étant créé, il s'agissait de le mettre à l'épreuve. Le pré-test de l'instrument a été réalisé au printemps 1974. À ce moment, chaque éducateur du quartier a évalué chacun des garçons présents. Les 61 pensionnaires de Boscoville ont été évalués par un minimum de deux éducateurs de leur quartier ( 8 jeunes sur 61 ) et par un maximum de six éducateurs ; la grande majorité a été évaluée par au moins cinq éducateurs ( 35 jeunes sur 61).

Le contrôle de la qualité de l'instrument construit s'est fait à partir des vérifications suivantes :

- le contrôle de l'observabilité des structures représentées par les 36 indicateurs d'étapes : pour ce faire nous avons calculé la proportion des éducateurs qui étaient incapables de répondre quant à la présence ou à l'absence d'un indicateur;

- la vérification de la fidélité des indicateurs à partir de l'évaluation du consensus entre les éducateurs de chaque quartier pour chacun des jeunes;

- la vérification de la structure interne des trois échelles (pairs, activités, éducateurs) et de sa correspondance avec le modele théorique comprenant trois étapes et douze phases. Cette vérification a été réalisée à l'aide de la méthode de Guttman;

2. L'équipe se composait de Bernard Tessier, Michèle Devroede-Melançon et Hélène Déom.

3. Le comité était composé de Guy Bélanger, Maurice Cusson, JeanPaul Déom, Jean Ducharme, Jeanine Guindon, Bernard Tessier. 
— finalement, le contrôle de la validité de l'instrument a été assuré par l'analyse des corrélations entre les évaluations cliniques habituelles et les résultations de l'instrument construit.

\section{LES RÉSULTATS}

\section{L'observabilité}

La conceptualisation de l'instrument pose que les indicateurs d'étapes doivent référer à des conduites observables, c'est-à-dire que les éducateurs soient capables de statuer, pour chaque jeune, sur la présence et l'absence de l'indicateur et que la présence des indicateurs soit substantielle dans la population à l'étude. Le tableau I rapporte ces résultats.

II ressort de l'analyse du tableau I que les éducateurs sont capables de coter les jeunes sur chacun des indicateurs d'étapes. En effet, seulement $3 \%$ des éducateurs sont en général incapables d'évaluer la présence d'un indicateur d'étapes; avec un minimum de $0 \%$ et un maximum de $8 \%$. De plus, on n'observe aucune concentration d'incapacité à évaluer les jeunes sur certaines étapes ou phases, dans l'une ou l'autre des situations d'évaluation.

Si l'on regarde la façon dont les jeunes ont été cotés, on observe qu'en moyenne $47 \%$ des sujets possèdent les indicateurs mais que cette proportion, quelle que soit la situation (pairs, éducateurs, activités), passe d'environ $90 \%$ à l'étape acclimatation, à $60 \%$ à $70 \%$ à l'étape contrôle, à $30 \%$ à $50 \%$ à l'étape production et finalement à $10 \%$ pour l'étape personnalité. Cette répartition des jeunes correspond bien aux attentes que nous suggérait la théorie.

En somme, les résultats présentés au tableau I nous permettent d'affirmer que les indicateurs d'étapes cernent réellement des comportements observables.

\section{La fidélité des énoncés}

La fidélité d'un instrument réfère à l'absence relative d'erreur de mesure ; en conséquence, pour être fidèle, un instrument doit être constant, c'est-à-dire nous assurer qu'il y a concordance entre les lectures sur un même individu à un même moment. Puisque plusieurs éducateurs évaluaient chacun des jeunes, nous 
TABLEAU I

Observabilité et fidélité des indicateurs d'étapes

\begin{tabular}{|c|c|c|c|c|}
\hline & & $\begin{array}{l}\text { Consensus } \\
\text { entre } \\
\text { éducateurs }\end{array}$ & $\begin{array}{c}\text { Observabilité } \\
\text { par } \\
\text { educateur }\end{array}$ & $\begin{array}{c}\text { Proportions } \\
\text { de sujets } \\
\text { ayant l'indicateur }\end{array}$ \\
\hline \multicolumn{2}{|l|}{ ACTIVITES } & & & \\
\hline Acclimatation & $1^{1 *}$ & $\begin{array}{l}, 96 \\
, 91 \\
, 83\end{array}$ & $\begin{array}{l}, 00 \\
, 00 \\
, 03\end{array}$ & $\begin{array}{l}97 \\
, 93 \\
, 69\end{array}$ \\
\hline Contrôle & $\begin{array}{l}4 \\
5 \\
6\end{array}$ & $\begin{array}{l}, 80 \\
, 78 \\
, 79\end{array}$ & $\begin{array}{l}, 08 \\
, 03 \\
, 03\end{array}$ & $\begin{array}{l}47 \\
, 51 \\
, 49\end{array}$ \\
\hline Production & $\begin{array}{l}7 \\
8 \\
9\end{array}$ & $\begin{array}{l}, 81 \\
, 83 \\
, 84\end{array}$ & $\begin{array}{l}02 \\
, 00 \\
, 03\end{array}$ & $\begin{array}{l}, 75 \\
, 33 \\
, 26\end{array}$ \\
\hline Personnalité & $\begin{array}{l}10 \\
11 \\
12\end{array}$ & $\begin{array}{l}, 83 \\
, 92 \\
, 88\end{array}$ & $\begin{array}{l}, 03 \\
, 05 \\
, 07\end{array}$ & $\begin{array}{l}18 \\
, 10 \\
, 08\end{array}$ \\
\hline \multicolumn{5}{|c|}{ EDUCATEURS } \\
\hline Acclimatation & $\begin{array}{l}13 \\
14 \\
15\end{array}$ & $\begin{array}{l}, 86 \\
, 75 \\
, 96\end{array}$ & $\begin{array}{l}, 03 \\
, 05 \\
, 00\end{array}$ & $\begin{array}{r}, 92 \\
, 72 \\
1,00\end{array}$ \\
\hline Contrôle & $\begin{array}{l}16 \\
17 \\
18\end{array}$ & $\begin{array}{l}, 87 \\
, 79 \\
, 77\end{array}$ & $\begin{array}{l}, 02 \\
, 02 \\
, 07\end{array}$ & $\begin{array}{l}.85 \\
.72 \\
, 41\end{array}$ \\
\hline Production & $\begin{array}{l}19 \\
20 \\
21\end{array}$ & $\begin{array}{l}, 79 \\
, 82 \\
, 77\end{array}$ & $\begin{array}{l}, 02 \\
, 08 \\
, 05\end{array}$ & $\begin{array}{l}, 59 \\
, 23 \\
, 59\end{array}$ \\
\hline Personnalité & $\begin{array}{l}22 \\
23 \\
24\end{array}$ & $\begin{array}{l}, 87 \\
, 89 \\
, 85\end{array}$ & $\begin{array}{l}, 03 \\
, 02 \\
, 02\end{array}$ & $\begin{array}{l}18 \\
, 20 \\
, 13\end{array}$ \\
\hline \multicolumn{5}{|l|}{ PAIRS } \\
\hline Acclimatation & $\begin{array}{l}25 \\
26 \\
27\end{array}$ & $\begin{array}{l}, 93 \\
, 80 \\
, 81\end{array}$ & $\begin{array}{l}, 02 \\
, 02 \\
, 03\end{array}$ & $\begin{array}{l}97 \\
, 57 \\
, 75\end{array}$ \\
\hline Contrôle & $\begin{array}{l}28 \\
29 \\
30\end{array}$ & $\begin{array}{l}, 82 \\
, 85 \\
, 90\end{array}$ & $\begin{array}{l}, 03 \\
, 03 \\
, 02\end{array}$ & $\begin{array}{l}56 \\
, 15 \\
, 12\end{array}$ \\
\hline Production & $\begin{array}{l}31 \\
32 \\
33\end{array}$ & $\begin{array}{l}, 75 \\
, 84 \\
, 87\end{array}$ & $\begin{array}{l}, 05 \\
, 02 \\
, 02\end{array}$ & $\begin{array}{l}, 57 \\
, 33 \\
, 31\end{array}$ \\
\hline Personnalité & $\begin{array}{l}34 \\
35 \\
36\end{array}$ & $\begin{array}{l}91 \\
, 87 \\
, 89\end{array}$ & $\begin{array}{l}, 02 \\
, 02 \\
, 03\end{array}$ & $\begin{array}{l}15 \\
, 15 \\
, 13\end{array}$ \\
\hline \multicolumn{2}{|c|}{ Moyenne générale } &, 85 & ,09 &, 47 \\
\hline
\end{tabular}

* Ces chiffres correspondent aux indicateurs détaillés à l'annexe. 
étions en bonne position pour évaluer le consensus entre les éducateurs.

Le tableau I rapporte la proportion moyenne de consensus entre les éducateurs pour chacun des 36 indicateurs d'étapes. Cette proportion a été calculée de la façon suivante : pour chacun des indicateurs d'étapes et pour chaque quartier nous avons calculé l'accord, quant à la présence de l'indicateur et ceci pour chacun des jeunes; par la suite, nous avons calculé la moyenne du consensus pour chaque quartier et finalement pour l'ensemble de Boscoville.

Le consensus moyen pour l'ensemble des indicateurs et l'ensemble des quartiers est de ,85, ce qui constitue un indice de fidélité tout à fait excellent. Cette conclusion s'appuie sur le fait qu'on exige habituellement ,80 (voir LeBlanc, 1971) et que dans le cas des indicateurs d'étapes on tenait compte de trois situations différentes (pairs, éducateurs et activités), de 12 indicateurs par situation et de sept équipes différentes d'éducateurs.

Le consensus moyen (tableau I) varie de ,75 à ,96. Tandis que pour les activités il est de ,86 (min. : ,78 et max. : ,96) contre ,83 pour les relations avec les éducateurs (min. : ,75 et max. : ,96) et ,85 pour les relations avec les pairs (min. : ,75 et max. : ,93). Par ailleurs, nous avons noté que quelle que soit la situation d'évaluation, le consensus était toujours plus fort pour les indicateurs des étapes acclimatation et personnalité et plus faible pour les étapes du milieu : production et contrôle. Dans le premier cas le consensus est supérieur à ,80, sinon supérieur à, 90 , tandis que dans le second cas, on observe des indicateurs avec un consensus entre, 75 et, 80 .

En somme, les données rapportées nous permettent d'affirmer que les indicateurs d'étapes sont des mesures fidèles de l'état des jeunes dans leurs relations avec les pairs et les éducateurs et de leur évolution dans les activités. Nous pouvons donc affirmer que les 36 indicateurs d'êtapes mesurent bien quelque chose, et ceci d'une qualité assez égale d'un indicateur à l'autre.

\section{La structure interne des échelles}

Chaque situation d'observation (pairs, éducateurs, activités) constituant des contextes de vie relativement indépendants, nous avons donc opté pour la construction de trois échelles permettant 
de situer l'évolution des jeunes. Chaque échelle, de par la perspective théorique énoncée précédemment doit avoir une structure interne qui soit en accord avec les principes suivants : les acquisitions se maintiennent à mesure que l'individu progresse et il y a un ordre spécifique entre les phases d'évolution. Ces principes signifient que pour chaque situation d'observation ou échelle les indicateurs d'étapes doivent apparaître chez le jeune dans une séquence spécifique et qu'une fois que l'indicateur est présent, il sera définitivement acquis.

Cette formulation de la structure interne des échelles correspond à l'échelle dite de Guttman (Guttman, 1950; Edwards, 1957 ; Maranell, 1974 ; Summers, 1970) et Wohlwill (1973) la recommande pour vérifier une séquence postulée de stades. Les avantages de cette technique sont la confrontation de l'échelle théorique et de l'échelle empirique et de la découverte de l'ordonnancement le plus efficace des indicateurs d'une échelle. Nous avons appliqué la technique de Guttman aux 12 indicateurs se rapportant aux relations avec les pairs, à ceux traitant des relations avec les éducateurs et à ceux référant à la performance dans les activités.

En ce qui concerne l'évolution dans les activités, on peut affirmer que la structure interne des douze item composant l'échelle activité est conforme aux principes du maintien et de la commulativité des acquis. En effet, il est possible de construire une échelle Guttman de douze énoncés car le coefficient de reproductibilité est de ,91 et l'amélioration sur l'échelle est de $17 \%$ par rapport au coefficient minimum de reproductibilité qui est de ,74. Le coefficient d'échellonnement étant de ,65, on peut donc affirmer que cette échelle est unidimensionnelle et cumulative. De plus, nous avons vérifié la possibilité que le coefficient de reproductibilité puisse être dû au hasard ; en utilisant la procédure de Schuessler (1974), nous avons démontré qu'il n'en était rien $(\mathrm{z}=15,29, \mathrm{p}<, 00001)$.

En conséquence de ces résultats, nous pouvons conclure que pour les activités, la théorie des étapes de la rééducation se vérifie dans le cadre de l'opérationnalisation que nous présentons. Par ailleurs, l'ordre des douze phases n'est pas celui attendu comme le montre la figure I. Il y apparaît que les phases 1, 2, 5, 6 et 8 à 12 présentent un ordre correspondant parfait entre leur position théorique et leur position empirique. La différence entre 
l'échelle postulée et celle observée vient de l'indicateur 7. Cette situation peut conduire à deux interprétations: la position de l'indicateur 7 a été mal conçue initialement ou cet énoncé a été mal formulé. La première alternative est peu probable car si nous forçons la position de l'indicateur 7 sur l'échelle, il n'est pas possible de constituer une échelle, ni une quasi-échelle. En effet, dans ce cas le coefficient de reproductibilité est inférieur à ,90, ,86 et puisque l'erreur ne se distribue pas au hasard sur les patrons a-typiques de réponse (voir LeBlanc et Tessier, 1975).

FIGURE I

Indicateurs d'étapes: leur séquence

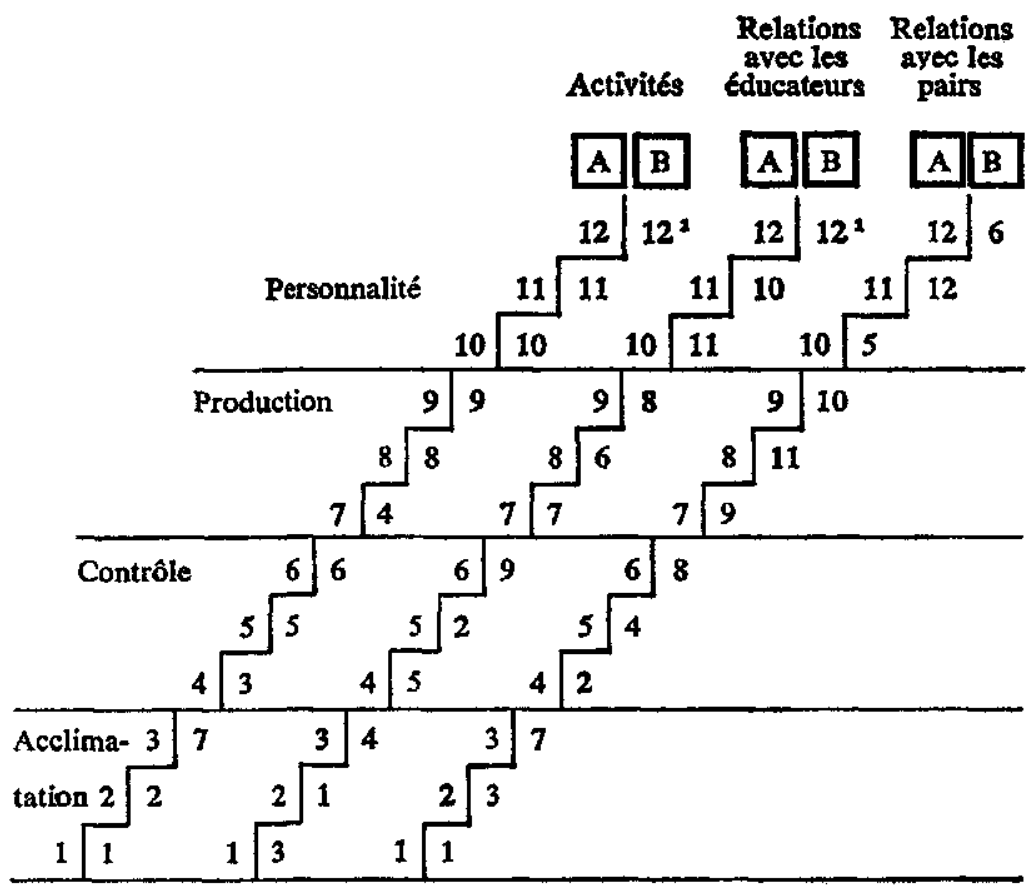

rép. : ,90 rê̆p. : , 89 rép. : ,89

scal. : ,65 scal. : ,50 scal. : ,53

1 Voir l'annexe pour le contenu des énoncés.

Echelle telle que définie théoriquement.

Echelle observée empiriquement. 
On peut donc conclure qu'il y a une séquence précise des acquisitions dans les activités et on confirme ainsi la théorie des étapes de la rééducation pour cette sphère. Cette conclusion est d'autant plus forte que les éducateurs observaient des activités différentes (boulot, sports, ...) et que la cote du jeune, quant à la présence ou l'absence de l'acquisition, dépendait de l'accord entre les éducateurs.

Pour les relations avec les pairs et avec les éducateurs une séquence théorique de douze phases était aussi postulée mais les vérifications statistiques n'ont pas produit des résultats aussi positifs que pour la performance dans les activités. En effet, le coefficient de reproductibilité est très légèrement inférieur au minimum requis pour ces deux échelles : pour les relations avec les pairs, il est de ,89 et de même pour les relations avec les éducateurs. Puisque ces valeurs sont supérieures à ,85, on peut conclure à l'existence de ce qu'Edwards (1957) appelle une quasiéchelle. La figure I met en parallèle l'ordre théorique et la séquence empirique des énoncés. On y observe des différences énormes entre l'ordre théorique postulé et la séquence empirique observée.

Les échelles de relations avec les pairs et avec les éducateurs ne sont donc pas des échelles de plein droit mais elles sont des quasi-échelles. Toutefois, il convient d'être sceptique à l'égard de cette conclusion parce que l'échelle relation avec les pairs contient deux patrons a-typiques avec $6 \%$ d'erreurs et l'echelle de relations avec les pairs en compte un avec $6 \%$ des erreurs (voir LeBlanc et Tessier, 1975).

Compte tenu de ces faits et parce que la validation finale viendra avec les données longitudinales, nous considérerons ces échelles comme des quasi-échelles. En somme, il s'avère impossible de confirmer complètement, et sans doute aucun, la théorie des étapes de la rééducation dans le cadre des relations des jeunes avec leurs pairs et avec les éducateurs.

Deux explications peuvent être avancées en rapport avec cette conclusion. On peut imputer l'échec partiel, dans la construction d'échelles respectant les postulats des acquis cumulatifs, à l'opérationnalisation de la théorie, c'est-à-dire la sélection et la formulation des indicateurs. Compte tenu de la démarche décrite précédemment pour sélectionner et formuler les indicateurs d'étapes, nous sommes enclins à rejeter cette explication. Il nous 
semble plus adéquat de retenir l'explication suivante de notre échec relatif. En ce qui concerne les relations aveć les pairs et avec les éducateurs, par leur nature même, on ne fait pas appel à un processus d'apprentissage séquentiel proprement dit ; à tout le moins, on peut penser qu'il ne s'agit pas du même type d'apprentissage que dans les activités. En conséquence, il serait plus normal de ne pas confirmer clairement le modèle théorique du processus de rééducation dans les sphères des relations avec les éducateurs et les pairs. Par ailleurs, une lecture de la tradition de Boscoville et une relecture du livre de Guindon (1970), nous portent à penser que la théorie est beaucoup moins documentée et beaucoup moins approfondie dans ces sphères que dans celle des activités. Tessier (1971) reconnaît aussi cette possibilité.

En résumé, la structure interne de l'échelle activités est tout à fait conforme au modèle théorique du processus de rééducation, mais nous ne pouvons poser un jugement totalement positif, ni entièrement négatif, à cet égard, pour les échelles de relations avec les pairs et de relations avec les éducateurs.

\section{La validité des échelles}

Nous avons construit trois échelles. Une excellente : la performance dans les activités, et deux de moindre qualité : les relations avec les pairs et avec les éducateurs. Voyons maintenant si ces échelles sont valides. Mesurent-elles quelque chose? La réponse à cette question est obtenue en vérifiant s'il y a un accord entre les résultats sur nos échelles et le jugement clinique des éducateurs, jugement qui porte sur la progression des individus dans le processus de rééducation.

A Boscoville, l'évaluation de la progression des jeunes, suivant les étapes de rééducation, est faite à partir de la compétence réelle, les acquisitions telles que théoriquement définies, et de la compétence mathématique qui dépend de la conformité aux attentes de comportements (voir Beaulne, 1974, pour plus de détails à ce sujet). Puisqu'il y a un coefficient d'association $\mathrm{de}, 89\left(\mathrm{Tau}_{\mathrm{b}}\right)$ entre ces deux mesures, nous ne nous servions que de la compétence réelle.

Pour contrôler si les trois échelles construites étaient valides, nous avons demandé aux éducateurs une évaluation globale de chaque jeune sur l'étape atteinte sur les plans des pairs, des éducateurs et des activités. Le consensus moyen entre les édu- 
TABLEAU II

Etapes de réducation: mesures d'associations

Compétence réelle : intercorrélations

\begin{tabular}{|l|c|c|c|c|}
\hline & Pairs & Educateurs & Activités & $\begin{array}{c}\text { Correlations } \\
\text { moyennes }\end{array}$ \\
\hline Pairs & &, $93^{2}$ &, 87 &, 90 \\
\hline Educateurs & & &, 89 &, 91 \\
\hline Activites & & & &, 88 \\
\hline
\end{tabular}

1 $\mathbf{T a u}_{\mathrm{b}}$

Indicateurs d'étapes : intercorrélations

\begin{tabular}{|l|c|c|c|c|}
\hline & Pairs & Educateurs & Activités & $\begin{array}{c}\text { Correlations } \\
\text { moyennes }\end{array}$ \\
\hline Pairs & &, $59^{1}$ &, 61 &, 60 \\
\hline Educateurs & & &, 58 &, 59 \\
\hline Activités & & & &, 60 \\
\hline
\end{tabular}

$1 \mathrm{Tau}_{\mathrm{b}}$

Intercorrélations des indicateurs d'étapes et des évaluations cliniques

\begin{tabular}{|l|c|c|c|c|}
\hline $\begin{array}{l}\text { Evaluation clinique } \\
\text { Indicateurs d'étapes }\end{array}$ & Pairs & Educateurs & Activités & $\begin{array}{c}\text { Corrélations } \\
\text { moyennes }\end{array}$ \\
\hline Pairs &, 62 &, 60 &, $56^{2}$ &, 59 \\
\hline Educateurs &, 44 &, 44 &, 44 &, 42 \\
\hline Activités &, 60 &, 59 &, 60 &, 60 \\
\hline
\end{tabular}

$2 \operatorname{Tan}_{b}$ 
cateurs pour un même jeune permet de situer chaque jeune sur les étapes du processus de rééducation. Nous avons noté (tableau II) qu'il y a une forte redondance entre les évaluations cliniques, puisque les associations entre les trois mesures de compétence réelles varient entre, 93 et, 87 , ce qui est très élevé. Pour leur part, les indicateurs d'étapes sont beaucoup plus indépendants, les associations varient de ,58 à ,61. Ces dernières associations signalent une certaine indépendance mais aussi que ces mesures se rapportent au même thème: le processus de rééducation.

En somme, les évaluations cliniques sur la performance dans les activités et les relations avec les pairs et les éducateurs se contaminent l'une l'autre tandis que les échelles d'indicateurs d'étapes mesurent des aspects différents du processus de rééducation.

Le tableau II nous permet aussi d'affirmer que nos mesures, les indicateurs d'étapes sur les plans des pairs, des éducateurs et des activités, sont valides car les associations, avec une mesure différente du même phénomène, varient de, 44 pour les relations avec les éducateurs à , 60 pour la performance dans les activités et à ,62 pour les relations avec les pairs.

Donc les échelles construites mesurent bien ce qu'elles sont censées mesurer et elles permettent de situer le jeune dans le processus de rééducation.

Pour terminer la vérification de la validité des indicateurs d'étapes, nous avons établi l'étape générale de rééducation atteinte à partir des échelles que nous avons construites (voir LeBlanc et Tessier, 1975), que nous avons correlées avec l'évaluation clinique de l'étape réelle atteinte.

La corrélation, entre l'évaluation clinique et l'évaluation de l'évolution des jeunes suivant les mesures que nous avons construites, est de ,62 $\left(\mathrm{Tau}_{\mathfrak{b}}\right)$. Elle nous indique donc que l'instrument construit des indicateurs d'étapes est valide et que cet instrument n'est pas redondant avec l'évaluation clinique. En effet, l'association est élevée mais non excessive.

Le tableau III, pour sa part, nous permet de constater que l'écart entre l'évaluation clinique et les indicateurs d'étapes est assez constant, sauf que l'évaluation clinique situe en acclimatation beaucoup de jeunes qui sont placés en contrôle à l'aide des 
TABLEAU III

Etapes de rééducation: évaluation clinique et indicateurs d'étapes

\begin{tabular}{|c|c|c|c|c|c|}
\hline Evaluation clinique & \multirow{2}{*}{$\begin{array}{c}\text { Acclima- } \\
\text { tation }\end{array}$} & \multirow{2}{*}{$\begin{array}{l}\text { Con- } \\
\text { trôle }\end{array}$} & \multirow{2}{*}{$\begin{array}{l}\text { Produc- } \\
\text { tion }\end{array}$} & \multirow{2}{*}{$\begin{array}{l}\text { Person- } \\
\text { nalité }\end{array}$} & \multirow{2}{*}{ Total } \\
\hline Indicateurs d'étapes & & & & & \\
\hline Acclimatation & 5 & 2 & 1 & & $\begin{array}{c}8 \\
17,8 \%\end{array}$ \\
\hline Contrôle & 12 & 5 & 4 & & $\stackrel{21}{46,7 \%}$ \\
\hline Production & & & 7 & 3 & $\begin{array}{c}10 \\
22,2 \%\end{array}$ \\
\hline Personnalité & & & 3 & 3 & ${ }_{13,3}^{6} \%$ \\
\hline Total & $\begin{array}{c}17 \\
37,8 \%\end{array}$ & $\begin{array}{c}7 \\
15,6 \%\end{array}$ & ${ }_{33,3 \%}^{15}$ & $\stackrel{6}{13,3 \%}$ & $\begin{array}{l}45 \\
100 \%\end{array}$ \\
\hline
\end{tabular}

indicateurs d'étapes. Cette situation s'explique par le fait que l'évaluation clinique générale est redondante avec les évaluations cliniques sur les activités et les relations avec les pairs et les éducateurs. En effet, les corrélations sont très élevées (tableau III). Cette situation ne se retrouve pas pour les indicateurs d'étapes et en conséquence les décalages dont parlent les éducateurs (voir Beaulne, 1974) y apparaissent plus clairement.

En somme, les trois échelles construites sont valides et la mesure générale de progression dans la rééducation l'est aussi. Toutefois nous constatons empiriquement des décalages entre la progression sur l'une ou l'autre des trois sphères : pairs, éducateurs et activités. Si nous définissons le décalage comme la différence entre l'étape atteinte sur chacune des trois échelles (voir LeBlanc et Tessier, 1975), nous obtenons les résultats suivants :

$\begin{array}{ccc}\text { différence } & \text { nb. jeunes } & \% \\ 2 & 23 & 37,7 \% \\ 0 & 25 & 41 \% \\ -2 & 11 & 13 \% \\ -4 & 2 & 3,3 \%\end{array}$

Il apparaît qu'il n'y a aucun décalage dans $41 \%$ des cas et des décalages chez $59 \%$ des jeunes. Ce décalage par contre prend deux formes : premièrement la performance dans les acti- 
vités précède la progression dans les relations avec les pairs et les éducateurs et ceci d'une étape chez $37,7 \%$ des jeunes ; deuxièmement la performance dans les activités suit la progression dans les autres sphères, ceci chez $21,3 \%$ des jeunes dont $18 \%$ d'une étape et 3,3\% de deux étapes. En somme, chez les premiers sujets la performance dans les activités entraîne la progression dans les autres sphères et chez environ la moitié moins des jeunes l'inverse est vrai.

\section{CONCLUSIONS}

Cet article constitue une première étape dans la construction de mesures de l'évolution des jeunes au cours du processus de rééducation; il s'agit de la démarche transversale. Seule la démarche longitudinale permettra de conclure de façon définitive sur la valeur des échelles construites.

A l'heure actuelle, il est possible de conclure :

- que les indicateurs d'étapes de rééducation, sélectionnés et formulés, sont des conduites observables de la part des éducateurs ;

- que les indicateurs construits, pris individuellement, sont fidèles car il y a peu de différence entre les éducateurs qui évaluent un même sujet ;

- que l'échelle performance dans les activités est la seule qui corresponde parfaitement au modèle théorique des étapes de la rééducation; les autres échelles; relations avec les pairs et avec les éducateurs, s'en approchent mais pas parfaitement ;

- que les trois échelles construites apparaissent valides car les associations avec les mesures indépendantes sont fortes ;

— qu'il est possible d'évaluer précisément les décalages entre les échelles se rapportant à la performance dans les activités et les relations avec les éducateurs et les pairs.

À la lecture de ces résultats, nous pouvons conclure qu'il est définitivement possible de construire un instrument rapide, simple et systématique qui permette de situer les jeunes en regard des quatre étapes du processus de rééducation. Toutefois, cet instrument ne remplacera jamais des données cliniques en profondeur mais il ne sera toujours qu'un point de départ pertinent pour la discussion de cas. 


\section{ANNEXE \\ Les indicateurs d'etapes}

Activité :

1. En activité, sous la surveillance constante de l'éducateur, il peut débuter son travail au signal donné ; n'utiliser que l'espace qui lui est alloué; suivre la procédure et compléter le travail prévu par le programme pour chaque période.

2. En activité, sous la surveillance constante de l'éducateur, il peut débuter son travail au signal donné. Ensuite, de lui-même, il peut n'utiliser que l'espace qui lui est alloué ; suivre la procédure et compléter le travail prévu par le programme pour chaque période.

3. En activité, il peut de lui-même, débuter son travail au signal donné; n'utiliser que l'espace qui lui est alloué ; suivre la procédure et compléter le travail prévu par le programme.

4. En activité, il peut choisir un instrument, une technique ou une procédure pour atteindre dans le temps prévu et l'espace mis à sa disposition, le but déterminé par le programme.

5. En activité, lorsqu'il a atteint le but déterminé par le programme dans le temps prévo et l'espace alloué, il peut retracer les techniques, les instruments ou les procédures qu'il a utilisés.

6. En activité, lorsqu'il a atteint le but déterminé par le programme, dans le temps prévu et l'espace alloué, il peut apprécier que c'est grâce à la technique, à l'instrument ou à la procédure qu'il a choisi.

7. En activité, avec un support de l'éducateur, il peut planifier la distribution de son travail dans le temps. Il peut utiliser de lui-même les moyens mis à sa disposition et réaliser approximativement ses prévisions.

8. En activité, de Iui-même, il peut planifier la distribution de son travail dans le temps, utiliser les moyens mis à sa disposition et réaliser ses prévisions tout en obtenant des résultats en accord avec ses capacités.

9. En activité, de lui-même, il peut planifier la distribution de son travail dans le temps, utiliser les moyens mis à sa distribution et obtenir des résultats en accord avec ses capacités, tout en tenant compte des besoins et des exigences du groupe.

10. En activité, il peut planifier son travail en fonction de ses aptitudes et des buts généraux qu'il veut atteindre lors de sa réinsertion sociale. Son application et sa façon personnelle de travailler sont constantes et indépendantes de l'atmosphère du groupe.

11. Il peut relier la planification et la réalisation de son travail en activité avec le style de vie personnel qu'il désire avoir lors de sa réinsertion sociale.

12. Il peut relier la planification et la réalisation de son travail en activité aux valeurs personnelles qu'il recherche dans sa réinsertion sociale.

\section{Educateur :}

1. Lorsque l'éducateur lui indique comment procéder dans les diverses situations de vie, il peut suivre les procédures transmises et accepter la supervision sans manifester ouvertement et évidemment sa désapprobation.

2. Il peut demander de lui-même à l'éducateur comment procéder dans diverses situations de vie et rechercher dans sa supervision la confirmation qu'il procède correctement. 
3. Lorsque l'éducateur est présent dans une situation de vie, il peut participer à l'action en cours sans chercher à éviter sa présence et sans manifester évidemment son opposition.

4. Lorsque l'éducateur intervient pour qu'il choisisse les moyens appropriés pour atteindre les buts prévus par le programme de réeducation, il peut se conformer à ses exigences tout en conservant généralement une même qualité de relation.

5. Le garçon peut recourir à l'éducateur pour s'assurer de la justesse des moyens qu'il choisit pour atteindre les buts prévus par le programme de rééducation.

6. Le garçon peut recourir a l'€ducateur pour mieux comprendre son rôle personnel dans la poursuite des buts prévus par le programme de réducation.

7. Il peut collaborer avec tous les éducateurs qui lui demandent de planifier le déroulement de sa démarche personnelle, en fonction des buts prévus par le programme de rééducation.

8. Il peut rechercher de lui-même le support de tous les éducateurs pour planifier le déroulement de sa démarche personnelle de réducation en fonction des buts prévus par le programme.

9. Il peut collaborer spontanément et régulièrement avec tous les éducateurs, dans un rôle formel ou informel, dans les divers moments de vie du groupe en fonction des buts prévus par le programme de rééducation.

10. Il peut parler spontanément et facilement avec les éducateurs des buts généraux qu'il désire atteindre lors de sa réinsertion sociale et de leur réalisme.

11. Il peut parler spontanément et facilement avec les educateurs du style de vie personnel qu'il désire lors de sa réinsertion sociale.

12. Il peut parler spontanément et facilement avec les éducateurs des valeurs personnelles qu'il recherche dans sa réinsertion sociale.

Pairs:

1. Sous la surveillance constante de l'éducateur, il peut demander dans le même lieu ou se trouve le groupe, sans manifester d'une façon évidente une incapacité de côtoyer, soit le groupe ou soit certains de ses membres et en se comportant avec tous de la façon prescrite par le programme de rééducation.

2. Il peut demander dans le même lieu où se trouve le groupe, sans manifester d'une façon évidente une incapacité de côtoyer, soit le groupe ou soit certains de ses membres et en se comportant avec tous de la façon prescrite par le programme de rééducation, sans que la surveillance constante de l'éducateur soit nécessaire.

3. Dans les diverses situations de vie en groupe, il peut se mêler de lui-même à la conversation et aux occupations du groupe en se comportant avec tous de la façon prescrite par le programme de rééducation.

4. Il peut se tenir plus régulièrement avec certains membres du groupe qui poursuivent évidemment les buts déterminés par le programme de rééducation, tout en se comportant avec tous de la façon prescrite par les us et coutumes du groupe.

5. A certaines occasions, il peut exprimer qu'il choisit ses amis parmi les les membres du groupe qui poursuivent les buts définis par le programme de réducation. 
6. A certaines occasions, il peut mentionner que son atteinte des buts définis par le programme de rééducation est le résultat de son choix d'amis parmi ceux qui poursuivent aussi ces buts.

7. Dans ses différentes occupations, avec le support de l'éducateur, il peut s'associer régulièrement avec les membres du groupe les plus aptes à lui faciliter la sélection et l'utilisation des meilleurs moyens pour atteindre les buts déterminés par le programme.

8. Dans ses différentes occupations, sans le support de l'éducateur, il peut s'associer régulièrement et de lui-même avec les membres du groupe les plus aptes à lui faciliter la sélection et l'utilisation des meilleurs moyens pour atteindre les buts déterminés par le programme de réćducation.

9. Dans les diverses occupations du groupe, il peut avoir spontanément et régulièrement une influence évidente auprès de ses compagnons dans la sélection et l'utilisation des moyens les plus appropriés pour atteindre les buts déterminés par le programme de rééducation, soit dans le rôle formel ou informel.

10. Lorsque l'occasion le demande, il peut exprimer clairement devant le groupe la relation entre sa propre façon d'agir et les buts généraux qu'il veut atteindre lors de sa réinsertion sociale. Ses prises de position sont stables et indépendantes de l'attitude du groupe.

11. Lorsque l'occasion le demande, il peut exprimer clairement devant le groupe la relation entre sa propre façon d'agir et le style de vie personnel qu'il désire avoir lors de sa réinsertion sociale. Ses prises de position sont stables et indépendantes de l'attitude du groupe.

12. Lorsque l'occasion le demande, il peut exprimer clairement devant le groupe la relation entre sa propre façon d'agir et les valeurs personnelles qu'il recherche dans sa réinsertion sociale. Ses prises de position sont stables et indépendantes de l'attitude du groupe.

\section{BIBLIOGRAPHIE}

BEAULNE, A. (1974), le Système de cotation a Boscoville, Montréal, Groupe de recherche sur l'inadaptation juvénile.

CUSSON, M. (1974), la Resocialisation du jeune délinquant, Montréal, Presses de l'Université de Montréal.

EDWARDS, R.L. (1957), Techniques of attitudes scale construction, New York, Appleton Century Crofts.

GENDREAU, G. (1974), les Etapes de rééducation d'après l'expérience de Boscoville, Conférence présentée à l'Université de Montréal (inédit).

GUINDON, J. (1970), les Etapes de la rééducation, Paris, Fleurus.

GUTTMAN, L. (1950), \& The basis for scalegram analysis in S.A. Stouffer et al ». Measurement and prediction, Princeton. Princeton University Press, p. 60-90.

LEBLANC, M. (1971), Analyses quantitatives, Montréal, Ecole de criminologie.

LEBLANC, M., TESSIER, B. (1975), Indicateurs d'étapes, Evaluation de Boscoville, Rapport technique no 10. G.R.I.J., Université de Montréal, $55 \mathrm{p}$.

MARANELL, G.M. (1974), Scaling: a source book, Chicago: Aldine, III $\mathbf{H}^{\mathrm{e}}$ partie. 
RILEY, M.W. et al (1954), Sociological studies in scale analysis, New Brunswick, Rutguers University Press.

SUMMERS, G.F. (1970), Attitude measurement, Chicago, Rand McNally. TESSIER, B. (1970), l'Evaluation de la relation rééducative dans le processus de rééducation, Montréal, Boscoville.

TESSIER, B. (1971), Cahiers des procédures de travail clinique, Montréal, Boscoville.

TESSIER, B. (1974), The psycho-educative model in action, Montréal, Ecole de psycho-éducation, Université de Montréal, document de travail $n^{\circ} 41$.

WOHLWILL, J.F., (1974), The study of behavioral development, New York, Academic Press.

ZEMAN, Z. (1974), Toward the development of a reeducation index, Montréal, Groupe de recherche sur l'inadaptation juvénile. 